\title{
Disfagia en el paciente crítico. ¿Un problema ignorado?
}

\author{
Dysphagia in the critically ill patient: an ignored problem?
}

\author{
José Benito Parra Maldonado, ${ }^{*}$ Miguel Ángel Martínez Camacho, ${ }^{*}$ \\ Pía Carolina Gallardo Astorga, * María Boy Skipsey*
}

Citar como: Parra MJB, Martínez CMÁ, Gallardo APC, Boy SM. Disfagia en el paciente crítico. ¿Un problema ignorado? Acta Med GA. 2021; 19 (4): 566-567. https://dx.doi.org/10.35366/102550

El paciente en estado crítico puede presentar insuficiencia respiratoria de tipo hipoxémica, dificultad en la protección de la vía aérea, disminución en el estado de alerta, entre otras, y requerirá de ventilación mecánica invasiva por medio de la intubación endotraqueal (en ocasiones de manera prolongada), afectando diferentes estructuras del complejo orofaríngeo, tales como: atrofia muscular, debilidad, rigidez, edematización y alteración en la sensibilidad, afectando la deglución. ${ }^{1}$ Estas complicaciones pueden perdurar por semanas o meses dependiendo de cada caso y el grado de complejidad.

Durante la desvinculación del paciente crítico de la ventilación mecánica (VM), proceso que culmina en la extubación, debemos considerar las posibles alteraciones de la deglución conocida como disfagia postextubación (DPE), un trastorno frecuente en las unidades de cuidados intensivos $(\mathrm{UCl})$ que se asocia de forma significativa a mayor incidencia de neumonía, reintubación, mortalidad intrahospitalaria, desnutrición y deshidratación, costos hospitalarios y estancia hospitalaria prolongada, afectando la calidad de vida del paciente y familiares. ${ }^{2,3}$

La DPE se define como la incapacidad de transferir de forma segura (protección de la vía aérea) y eficaz (nutrición e hidratación adecuada) secreciones, alimentos y líquidos de la boca hacia el estómago por la alteración de una o más fases de la deglución secundaria al proce-

\footnotetext{
* Servicio de Rehabilitación de la Terapia Intensiva Respiratoria Reconvertida, Hospital General de México "Dr. Eduardo Liceaga", Ciudad de México.
}

Correspondencia:

Miguel Ángel Martínez Camacho

Correo electrónico: Iftmiguelangelmtz@gmail.com

Aceptado: 12-04-2021.

www.medigraphic.com/actamedica

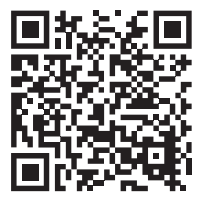

so de intubación, provocando una disincronía entre el patrón respiratorio con el patrón deglutorio. El riesgo de desarrollar DPE aumenta conforme la edad y los días de VM. Se considera como intubación prolongada aquélla que supera las 48 horas. $^{4,5}$

Existe un amplio rango en la incidencia de la DPE que varía entre un $3-62 \%$ considerando las posibles diferencias entre las poblaciones estudiadas, tipo de terapia intensiva, discrepancias en el diseño y métodos de estudio. 5,6 La DPE pudiera ser un trastorno subdiagnosticado ya que: 1) no hay protocolos establecidos en las $\mathrm{UCl}$; 2) las evaluaciones clínicas realizadas en la cabecera del paciente, aunque necesarias, no nos proporcionan información objetiva, como por ejemplo secreciones basales, movimiento cordal, penetración, aspiraciones silentes, entre otras; y 3) la falta de evaluaciones instrumentales en la $\mathrm{UCl}$.

La alimentación por vía oral se determinará con base en los resultados obtenidos en la evaluación instrumental debido a que es fundamental identificar consistencias y volúmenes adecuados para la alimentación segura y eficaz del paciente.

Se recomienda evaluar la deglución del paciente 24 horas postextubación, ${ }^{7}$ ya que se ha identificado que durante este periodo el paciente presenta edema, descoordinación, entre otras afectaciones que se pueden resolver. Existen diversas evaluaciones clínicas como el Test del vaso de agua, Gugging Swallowing Screen (GUSS), método de exploración clínica volumen viscosidad (MECV-V), entre otras que nos ayudan a identificar al paciente en riesgo de desarrollar DPE.

La evaluación de la deglución en el paciente crítico se deberá basar en la historia clínica, identificación de riesgos preexistentes y evaluación clínica e instrumental. El estándar de oro para diagnosticar la disfagia es mediante las evaluaciones instrumentales como la evaluación endoscópica de la deglución (FEES) y videofluoroscopia de la deglución (VFSE), pues permiten evaluar de manera 
objetiva las fases de la deglución y, en consecuencia, definir un plan de tratamiento puntual. ${ }^{6}$

Después del diagnóstico, se determinará vía de alimentación (oral, enteral, parenteral o más de una), se desarrollará un plan de tratamiento individualizado por un equipo multidisciplinario como foniatras, terapeutas de lenguaje y fisioterapeutas especializados en deglución que trabajarán la musculatura afectada previamente identificada.

El plan de rehabilitación puede contemplar diferentes ejercicios con los siguientes objetivos: 1) el fortalecimiento de la musculatura oral y faríngea; 2) tratamiento sensorial por la pérdida de la sensibilidad; 3) electroestimulación; y 4) maniobras que faciliten la deglución (previamente observadas en la evaluación instrumental).

Debido a lo anterior, es crucial la integración de profesionales especializados en deglución para el diagnóstico oportuno y rehabilitación adecuados con la finalidad de disminuir complicaciones, reducir costos y estancias hospitalarias.

\section{REFERENCIAS}

1. Brodsky MB, Gellar JE, Dinglas VD, Colantuoni E, Mendez-Tellez PA, Shanholtz C et al.. Duration of oral endotracheal intubation is associated with dysphagia symptoms in acute lung injury patients. J Crit Care. 2014; 29 (4): 574-579. doi: 10.1016/j.jcrc.2014.02.015.

2. Macht M, Wimbish T, Clark BJ, Benson AB, Burnham EL, Williams A et al. Postextubation dysphagia is persistent and associated with poor outcomes in survivors of critical illness. Crit Care. 2011; 15 (5): R231. doi: 10.1186/cc10472.

3. Brown CV, Hejl K, Mandaville AD, Chaney PE, Stevenson G, Smith C. Swallowing dysfunction after mechanical ventilation in trauma patients. J Crit Care. 2011; 26 (1): 108.e9-13. doi: 10.1016/j. jcrc.2010.05.036.

4. Rassameehiran S, Klomjit S, Mankongpaisarnrung C, Rakvit A. Postextubation dysphagia. Proc (Bayl Univ Med Cent). 2015; 28 (1): 18-20. doi: 10.1080/08998280.2015.11929174.

5. Skoretz SA, Flowers HL, Martino R. The incidence of dysphagia following endotracheal intubation: a systematic review. Chest. 2010; 137 (3): 665-673. doi: 10.1378/chest.09-1823.

6. Macht M, Wimbish T, Bodine C, Moss M. ICU-acquired swallowing disorders. Crit Care Med. 2013; 41 (10): 2396-2405. doi: 10.1097/ ccm.0b013e31829caf33.

7. Marvin S, Thibeault S, Ehlenbach WJ. Post-extubation dysphagia: does timing of evaluation matter? Dysphagia. 2019; 34 (2): 210-219. doi: 10.1007/s00455-018-9926-3. 